\title{
Risk of first and recurrent serious infection in sarcoidosis: a Swedish register-based cohort study
}

\author{
Marios Rossides (1) 1 , Susanna Kullberg ${ }^{2,3}$, Anders Eklund ${ }^{2,3}$, Daniela Di \\ Giuseppe ${ }^{1}$, Johan Grunewald ${ }^{2,3}$, Johan Askling ${ }^{1,4}$ and Elizabeth V. Arkema (1) ${ }^{1}$ \\ Affiliations: ${ }^{1}$ Clinical Epidemiology Division, Dept of Medicine Solna, Karolinska Institutet, Stockholm, \\ Sweden. ${ }^{2}$ Respiratory Medicine Division, Dept of Medicine Solna, Karolinska Institutet, Stockholm, Sweden. \\ ${ }^{3}$ Respiratory Medicine, Theme Inflammation and Infection, Karolinska University Hospital, Stockholm, \\ Sweden. "Rheumatology, Theme Inflammation and Infection, Karolinska University Hospital, Stockholm, \\ Sweden.
}

Correspondence: Marios Rossides, Karolinska Institutet, Dept of Medicine Solna, Clinical Epidemiology Division, Karolinska University Hospital T2, 17176 Stockholm, Sweden. E-mail: marios.rossidesaki.se

@ERSpublications

Sarcoidosis is associated with an increased risk of serious infections, especially during the first 2 years after diagnosis. Patients in need of immunosuppressants around diagnosis are twice as likely to develop serious infections than those who do not. https://bit.ly/2VFOvSo

Cite this article as: Rossides M, Kullberg S, Eklund A, et al. Risk of first and recurrent serious infection in sarcoidosis: a Swedish register-based cohort study. Eur Respir J 2020; 56: 2000767 [https://doi.org/10.1183/ 13993003.00767-2020].

ABSTRACT Serious infections impair quality of life and increase costs. Our aim was to determine if sarcoidosis is associated with a higher rate of serious infection and whether this varies by age, sex, time since diagnosis or treatment status around diagnosis.

We compared individuals with sarcoidosis (at least two International Classification of Diseases codes in the Swedish National Patient Register 2003-2013; n=8737) and general population comparators matched 10:1 on age, sex and residential location $(n=86376)$. Patients diagnosed in 2006-2013 who were dispensed at least one immunosuppressant \pm 3 months from diagnosis (Swedish Prescribed Drug Register) were identified. Cases and comparators were followed in the National Patient Register for hospitalisations for infection. Using Cox and flexible parametric models, we estimated adjusted hazard ratios (aHR) and 95\% confidence intervals for first and recurrent serious infections (new serious infection $>30$ days after previous).

We identified 895 first serious infections in sarcoidosis patients and 3881 in comparators. The rate of serious infection was increased 1.8-fold in sarcoidosis compared to the general population (aHR 1.81, 95\% CI 1.65-1.98). The aHR was higher in females than males and during the first 2 years of follow-up. Sarcoidosis cases treated with immunosuppressants around diagnosis had a three-fold increased risk, whereas nontreated patients had a $50 \%$ increased risk. The rate of serious infection recurrence was 2.8 -fold higher in cases than in comparators.

Serious infections are more common in sarcoidosis than in the general population, particularly during the first few years after diagnosis. Patients who need immunosuppressant treatment around diagnosis are twice as likely to develop a serious infection than those who do not.

This article has an editorial commentary: https://doi.org/10.1183/13993003.02376-2020

This article has supplementary material available from erj.ersjournals.com

Received: 19 March 2020 | Accepted after revision: 22 April 2020

Copyright OERS 2020. This version is distributed under the terms of the Creative Commons Attribution NonCommercial Licence 4.0. 


\section{Introduction}

Serious hospitalised infections ("serious infections") are associated with impaired quality of life and high costs, particularly if they are recurrent [1-4]. In addition, they contribute to the higher rate of premature death seen in sarcoidosis [4], but data on serious infection risks in sarcoidosis are limited. In other better-studied inflammatory diseases, serious infection risks are higher than in the general population and are often attributed to immunosuppressant treatment that is prescribed to almost all patients $[5,6]$. However, risks from other inflammatory diseases cannot be readily extrapolated to sarcoidosis because $\sim 60 \%$ of individuals with sarcoidosis do not need immunosuppressant treatment. Additionally, sarcoidosis resolves within $<5$ years from diagnosis in about half of patients [7].

In sarcoidosis, serious infection risks in comparison to the general population were investigated in only one study from the United States. In that study of 345 patients diagnosed between 1976 and 2013, a two-fold increased rate of serious infection compared to the general population was reported [8]. The rate ratio for serious infection was even higher (2.4) in patients who received immunosuppressants during the course of their disease [8]. It is unclear whether similar results can be obtained from contemporary and larger population-based sarcoidosis cohorts. Moreover, it remains uncertain which patients exhibit these perceived high risks for serious infection and whether they are at higher risk for developing recurrent serious infections. This information could prove particularly useful to properly target patient groups who can benefit from preventive interventions.

To answer these unresolved questions, large and longitudinal data are required. We therefore used Swedish register data to investigate serious infection in sarcoidosis. Our aim was to estimate relative risks of first and recurrent serious infection associated with sarcoidosis and examine if these varied by age at diagnosis, sex, treatment status around diagnosis, or time since diagnosis.

\section{Methods}

\section{Study setting and data sources}

In Sweden, the healthcare system is tax-funded and universally accessible to residents. Data generated by interaction with the healthcare system are captured in registers and can be linked using an individual's unique identification number. We created a large cohort of individuals with and without sarcoidosis by linking several nationwide and population-based registers. We collected information on hospitalisations and outpatient visits to specialist care from the National Patient Register (NPR; nationwide coverage since 1987 and 2001, on hospitalisations and outpatient visits, respectively). Data quality is high [9], but results of histological or imaging examinations are not available in the NPR. Prescription medication dispensations were obtained from the Prescribed Drug Register (PDR); these were available starting July 2005.

\section{Study population}

Using inpatient and outpatient visit data in the NPR, we identified all individuals who had two or more visits listing an International Classification of Diseases (ICD) code for sarcoidosis between January 1, 2003 and December 31, 2013. ICD codes are listed in supplementary table S1. Because most sarcoidosis cases are diagnosed in outpatient clinics [10], we allowed for 2 years of outpatient visit data to accumulate in the NPR so that we could capture newly diagnosed cases.

We further classified all individuals with sarcoidosis diagnosed starting January 1, 2006 into those treated or not for sarcoidosis around the time of diagnosis. Cases who were dispensed systemic corticosteroids, methotrexate or azathioprine within 3 months before or after the first visit for sarcoidosis (PDR data) were allocated to the treated group (supplementary table S1). We used treatment as an indicator of sarcoidosis severity around diagnosis $[4,7]$ and to study the role of treatment on the risk of serious infection.

At second visit for sarcoidosis, each case was matched on birth year, sex and residential location to up to 10 general population comparators sampled from the total population register, who had no history of sarcoidosis at the time. To reduce sarcoidosis misclassification, we excluded cases and comparators aged $<18$ or $>85$ years and those with a haematopoietic or lung malignancy recorded in the Swedish Cancer Register 6 months before or after the first visit for sarcoidosis or corresponding date for comparators (supplementary table S1).

Ethical permission was provided by the regional ethics review board in Stockholm (2014/230-31).

\section{Follow-up for first and recurrent serious infection}

The outcome, serious infection, was defined as a hospitalisation in the NPR listing an ICD code for an infectious disease (supplementary table S1). To minimise misclassification, infectious disease had to be the primary discharge diagnosis. We followed sarcoidosis cases and comparators from inclusion (second visit 
for sarcoidosis or corresponding date for comparators) to first admission for serious infection, death (Cause of Death Register), emigration (Total Population Register) or December 31, 2013, whichever occurred first. We identified the 10 most common diagnoses in cases and comparators and reported the frequency of serious opportunistic infections (i.e. aspergillosis, candidiasis, tuberculosis, other mycobacterial infections and pneumocystosis).

In addition, we examined recurrent unrelated serious infections by allowing for a serious infection to occur $>30$ days after the previous, irrespective of length of hospital stay. In both cases and comparators, occurrence of more than six serious infections was infrequent. To ensure statistical model stability, we allowed for only up to six recurrent serious infections per individual. To investigate the extent of differential loss to follow-up in the sarcoidosis and comparator groups, we identified deaths and deaths due to serious infection in the Cause of Death Register. For analyses of recurrent infections, follow-up started at inclusion and ended at the last serious infection (maximum six events per individual), death, emigration or December 31, 2013, whichever came first.

\section{Other variables}

Confounding variables were evaluated at baseline using data obtained from various registers (supplementary table S1). Briefly, we collected information on birth date (to calculate age), sex, birth country (grouped into Nordic and non-Nordic), residential location (grouped into six healthcare regions), civil status (married or in registered partnership, or other), years of education $(\leqslant 9,10-12$, $\geqslant 13$ years or missing) and salary earned the year before inclusion adjusted for 2014 inflation (www.statistikdatabasen. scb.se; SEK $0-<100000,100000-<300000,300000-<600000, \geqslant 600000$ or missing).

In addition, we approximated general health status at inclusion by counting the total number of inpatient or outpatient visits in the NPR within 2 years before the first sarcoidosis visit or corresponding date for comparators (grouped into $0,1-3, \geqslant 4$ visits). We also identified comorbidity associated with serious infections (autoimmune disease in study participants or first degree relatives (data from the Swedish Multi-Generation Register), primary immunodeficiency, stroke, diabetes, etc.) requiring at least one or at least two visits in the NPR or two or more dispensations in the PDR, as appropriate (supplementary table S1). To better describe baseline serious infection risk, we reported history of serious infection in the past year and dispensations of sarcoidosis treatments and antimicrobials (i.e. antibacterial, antimycobacterial, antifungal or antiviral medications) within 6 months before inclusion (supplementary table S1).

\section{Statistical analysis}

We estimated stabilised inverse probability of sarcoidosis weights that allowed us to obtain marginal adjusted estimates in subsequent analyses (details in the supplementary methods and table S2). Using weighted Poisson regression models, we estimated adjusted rates and rate differences for serious infections and their corresponding 95\% confidence intervals. To compare sarcoidosis to the general population, we used Cox models with follow-up years as the time scale and the weights to estimate adjusted hazard ratios (aHR). In addition, hazard ratios from unweighted models were reported. Because the risk of serious infection associated with sarcoidosis varied over time, therefore violating the Cox proportionality of hazards assumption, we used flexible parametric survival models [11] specified as described in the supplementary material. We plotted aHRs and marginal cumulative probabilities (risks) for sarcoidosis overall and by treatment status around diagnosis (treated/untreated).

We further stratified our analyses by age at inclusion (18-44, 45-64 or 65-85 years), sex, history of autoimmune disease and treatment status around diagnosis and examined modification of the aHRs by these factors using a likelihood ratio test. Because medication dispensation data became available mid-2005, analyses by treatment status were performed in a subset of the population (77\%) that entered the cohort starting 2006.

We modelled recurrent serious infections by adding a $\gamma$-frailty term to Cox and flexible parametric survival models to account for unobserved heterogeneity among individuals (refer to supplementary material for model specification).

In sensitivity analyses, we used a stricter definition for serious infection requiring at least one dispensation of an antimicrobial \pm 15 days of the hospital admission for serious infection to check whether misclassification of serious infection could affect our results. In addition, assuming pneumonias were more likely in sarcoidosis due to repeated lung imaging, we excluded pneumonias from the definition of serious infection in both sarcoidosis and comparators. Additionally, we disregarded urinary tract infections to assess whether hospital-acquired infections could explain the association. Furthermore, to examine how a lower threshold for hospitalisation for serious infection in sarcoidosis could affect our findings, we required comparators to have a visit in the NPR within 2 years before inclusion. Last, to test our results in the presence of sarcoidosis misclassification, we excluded cases and comparators with a history of serious 
infection within a year before the first sarcoidosis visit or the corresponding date for comparators and investigated serious infection in sarcoidosis cases diagnosed by pulmonologists at Karolinska University Hospital in Stockholm and registered in the local cohort.

Data were processed and analysed using SAS software version 9.4 (SAS Institute Inc., Cary, NC, USA) and $\mathrm{R}$ version 3.6.2 (R Core Team, R Foundation for Statistical Computing, Vienna, Austria).

\section{Results}

We compared 8737 sarcoidosis cases to 86376 matched general population comparators. Of cases diagnosed in 2006-2013, 41\% were treated with immunosuppressants around diagnosis. Cases and comparators were aged mean \pm SD $50 \pm 14.7$ years and $45 \%$ were female (table 1 ). At baseline, socioeconomic position was similar between the two groups, but history of morbidity, especially that of hypertension, diabetes and autoimmune disease was more prevalent in sarcoidosis cases. Cases were more likely to have a history of serious infection in the year before inclusion (4\% versus $1 \%$ ) and to have been dispensed an immunosuppressive or antimicrobial treatment in the previous 6 months.

After a median 4.8 years (95\% CI 4.8-4.9 years; similar in cases and comparators), we observed 895 first serious infections in sarcoidosis (rate 17.4, 95\% CI 16.0-18.9 per 1000 person-years) and 3881 serious infections in comparators (rate 9.6, 95\% CI 9.3-9.9 per 1000 person-years) (table 2). In both groups, the most common serious infection was pneumonia ( 25\%; supplementary table S3) and opportunistic serious infections were infrequent (supplementary table S4). Risk of serious infection by follow-up years are depicted in figure 1 and risks at 6 months, 5 and 10 years are summarised in supplementary figure S1.

TABLE 1 Demographic and clinical characteristics at inclusion of individuals with sarcoidosis and their matched general population comparators

\begin{tabular}{|c|c|c|}
\hline & Sarcoidosis & General population \\
\hline Subjects $n$ & 8737 & 86376 \\
\hline Age years & $49.8 \pm 14.8$ & $49.8 \pm 14.7$ \\
\hline Female & 44.5 & 44.6 \\
\hline Born in non-Nordic country" & 9.5 & 11.8 \\
\hline \multicolumn{3}{|l|}{ Education attained years } \\
\hline$\leqslant 9$ & 20.6 & 20.8 \\
\hline $10-12$ & 49.1 & 46.4 \\
\hline$\geqslant 13$ & 29.2 & 31.6 \\
\hline Missing & 1.2 & 1.2 \\
\hline \multicolumn{3}{|l|}{ History of comorbidity } \\
\hline Congestive heart disease & 2.4 & 1.3 \\
\hline Atrial fibrillation & 3.2 & 2.1 \\
\hline Acute myocardial infarction & 2.1 & 1.8 \\
\hline Stroke & 1.7 & 1.6 \\
\hline COPD & 2.3 & 1.0 \\
\hline Asthma & 4.6 & 2.4 \\
\hline Hypertension & 21.4 & 15.9 \\
\hline Diabetes mellitus & 7.5 & 4.2 \\
\hline Dyslipidaemia & 10.8 & 8.2 \\
\hline Autoimmune disease & 7.9 & 4.3 \\
\hline Primary immunodeficiency & 0.4 & 0.1 \\
\hline Serious infection in the past year & 3.9 & 0.8 \\
\hline$\geqslant 1$ medication dispensed in the past 6 months $s^{\pi}$ & $\mathrm{n}=6723$ & $\mathrm{n}=66441$ \\
\hline Systemic corticosteroids & 18.7 & 2.9 \\
\hline Other immunosuppressants ${ }^{+}$ & 1.2 & 0.7 \\
\hline Hydroxychloroquine & 0.1 & 0.1 \\
\hline Inhaled corticosteroids & 7.3 & 1.9 \\
\hline NSAIDs & 26.3 & 9.7 \\
\hline Antimicrobials ${ }^{\S}$ & 32.6 & 13.2 \\
\hline
\end{tabular}

Data are presented as mean \pm SD or $\%$, unless otherwise stated. Percentages may not sum to 100 owing to rounding. NSAIDs: nonsteroidal anti-inflammatory drugs. "\#: Nordic countries: Sweden, Denmark, Norway, Finland and Iceland (category excludes missing $<0.5 \%$ ); ${ }^{\text {: }}$ : ascertained in individuals who entered the cohort starting January 1, 2006 for whom medication dispensations could be obtained from the Prescribed Drug Register; ${ }^{+}$: includes methotrexate, azathioprine and leflunomide; ${ }^{\S}$ : includes antibacterial, antimycobacterial, antifungal and antiviral medications. 
TABLE 2 Rates, rate differences and hazard ratios (HRs) for first serious infection comparing sarcoidosis to the general population

\begin{tabular}{|c|c|c|c|c|c|c|}
\hline \multicolumn{2}{|r|}{ Sarcoidosis } & \multicolumn{2}{|c|}{ General population } & \multirow{2}{*}{$\begin{array}{c}\text { Adjusted rate } \\
\text { difference per } 1000 \\
\text { person-years } \\
(95 \% \mathrm{CI})\end{array}$} & \multirow{2}{*}{$\begin{array}{l}\text { Hazard ratio }{ }^{\#} \\
\qquad(95 \% \mathrm{CI}]\end{array}$} & \multirow{2}{*}{$\begin{array}{c}\text { Adjusted } \\
\text { hazard ratio } \\
(95 \% \mathrm{CI})\end{array}$} \\
\hline & $\begin{array}{c}\text { Adjusted rate per } \\
1000 \text { person-years }{ }^{\#} \\
(95 \% \mathrm{Cl})\end{array}$ & Events & $\begin{array}{c}\text { Adjusted rate per } \\
1000 \text { person-years } \\
(95 \% \mathrm{Cl})\end{array}$ & & & \\
\hline
\end{tabular}

\begin{tabular}{|c|c|c|c|c|c|c|c|}
\hline Subjects & & 8737 & & 86376 & & & \\
\hline Overall & 895 & $17.4(16.0-18.9)$ & 3881 & $9.6(9.3-9.9)$ & $7.8(6.3-9.2)$ & $2.40(2.23-2.58)$ & $1.81(1.65-1.98)$ \\
\hline \multicolumn{8}{|c|}{$\begin{array}{l}\text { Age at inclusion" } \\
\text { years }\end{array}$} \\
\hline $18-44$ & 222 & $8.8(7.4-10.5)$ & 937 & $5.1(4.8-5.4)$ & $3.7(2.2-5.3)$ & $2.39(2.07-2.77)$ & $1.74(1.44-2.09)$ \\
\hline $45-64$ & 327 & $16.1(14.0-18.5)$ & 1334 & $8.5(8.0-8.9)$ & $7.6(5.4-9.9)$ & $2.58(2.28-2.91)$ & $1.90(1.64-2.20)$ \\
\hline $65-85$ & 346 & $58.3(51.1-66.4)$ & 1610 & $27.0(25.7-28.3)$ & $31.3(23.5-39.1)$ & $2.45(2.18-2.75)$ & $2.16(1.88-2.49)$ \\
\hline \multicolumn{8}{|l|}{ Sex" } \\
\hline Female & 435 & 21.6 (19.2-24.3) & 1938 & $10.7(10.3-11.2)$ & $10.9(8.3-13.5)$ & $2.36(2.13-2.62)$ & $2.01(1.78-2.28)$ \\
\hline Male & 460 & $14.3(12.7-16.1)$ & 1943 & $8.7(8.3-9.1)$ & $5.6(3.8-7.4)$ & $2.45(2.21-2.71)$ & $1.64(1.45-1.87)$ \\
\hline \multicolumn{8}{|c|}{$\begin{array}{l}\text { Treatment status } \\
\text { around } \\
\text { diagnosis }\end{array}$} \\
\hline Treated & 326 & 29.8 (25.9-34.3) & 948 & $9.8(9.2-10.4)$ & 20.0 (15.8-24.2) & $3.70(3.26-4.19)$ & $3.04(2.61-3.55)$ \\
\hline Not treated & 275 & $15.7(13.5-18.3)$ & 1428 & $10.2(9.7-10.8)$ & $5.5(3.1-7.9)$ & $1.96(1.72-2.23)$ & $1.53(1.31-1.80)$ \\
\hline \multicolumn{8}{|c|}{ 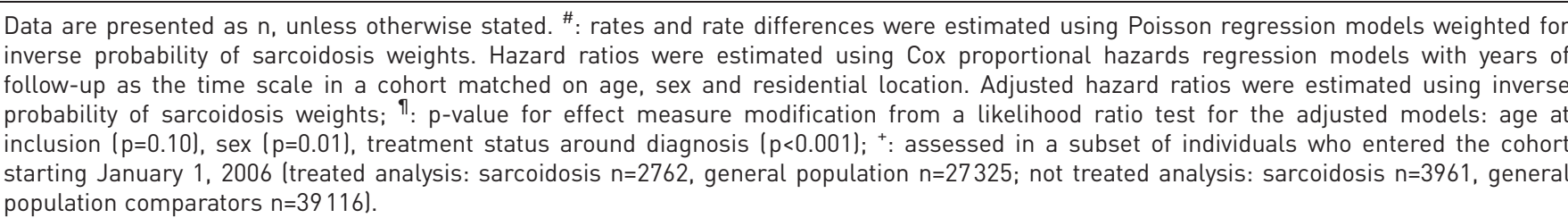 } \\
\hline
\end{tabular}

The aHR for serious infection comparing sarcoidosis to comparators was 1.81 (95\% CI $1.65-1.98$ ). However, there was notable variation by years of follow-up: the aHR was highest (increased approximately three-fold) around start of follow-up and decreased to $\sim 1.4$ within 2 years (figure 2 (aHRs) and supplementary figure S2 (rates)). The aHR for first serious infection was similar across age groups, but it was higher in females compared to males $(2.01$ versus $1.64, \mathrm{p}=0.01)$ and in individuals treated compared to those not receiving immunosuppressive treatment around diagnosis (3.04 versus $1.53, \mathrm{p}<0.001$; table 2 ).

Recurrent serious infections were twice as likely in sarcoidosis compared to the general population (within-individual aHR 2.79, 95\% CI 2.51-3.10; table 3) with variation of the aHR during follow-up (supplementary figure S3).

In sensitivity analyses depicted in table 4 , the aHR did not change considerably when pneumonias or urinary tract infections were disregarded or when individuals with a history of serious infection in the past year were excluded. However, it increased slightly when an antimicrobial medication dispensation was required around the time of admission for serious infection (aHR 2.23, 95\% CI 1.96-2.54). The association weakened when we required comparators to have a NPR visit within 2 years before inclusion (aHR for overall sarcoidosis 1.23, 95\% CI 1.12-1.35; treated and untreated sarcoidosis aHR 2.08 and 1.03, respectively). Lastly, we observed an aHR for serious infection of 1.49 (95\% CI 1.02-2.18) in the sarcoidosis cohort at Karolinska.

\section{Discussion}

In this large nationwide investigation, we found that sarcoidosis was associated with an overall 1.8-fold increased rate of first serious hospitalised infection. Serious infections occurred at a higher rate during the first 2 years since sarcoidosis diagnosis compared to the rest of follow-up. In individuals treated with immunosuppressants around the time of sarcoidosis diagnosis, the risk of serious infection was double the risk of those who did not receive treatment. Compared to the general population, individuals with sarcoidosis were more likely to develop multiple serious infections.

Our main finding of an almost two-fold increased rate of serious infection in sarcoidosis compared to the general population is in line with a report from the United States [8]. In that cohort of 345 primarily white American patients, investigators observed similar 10-year risks and rate ratios for serious infection to 


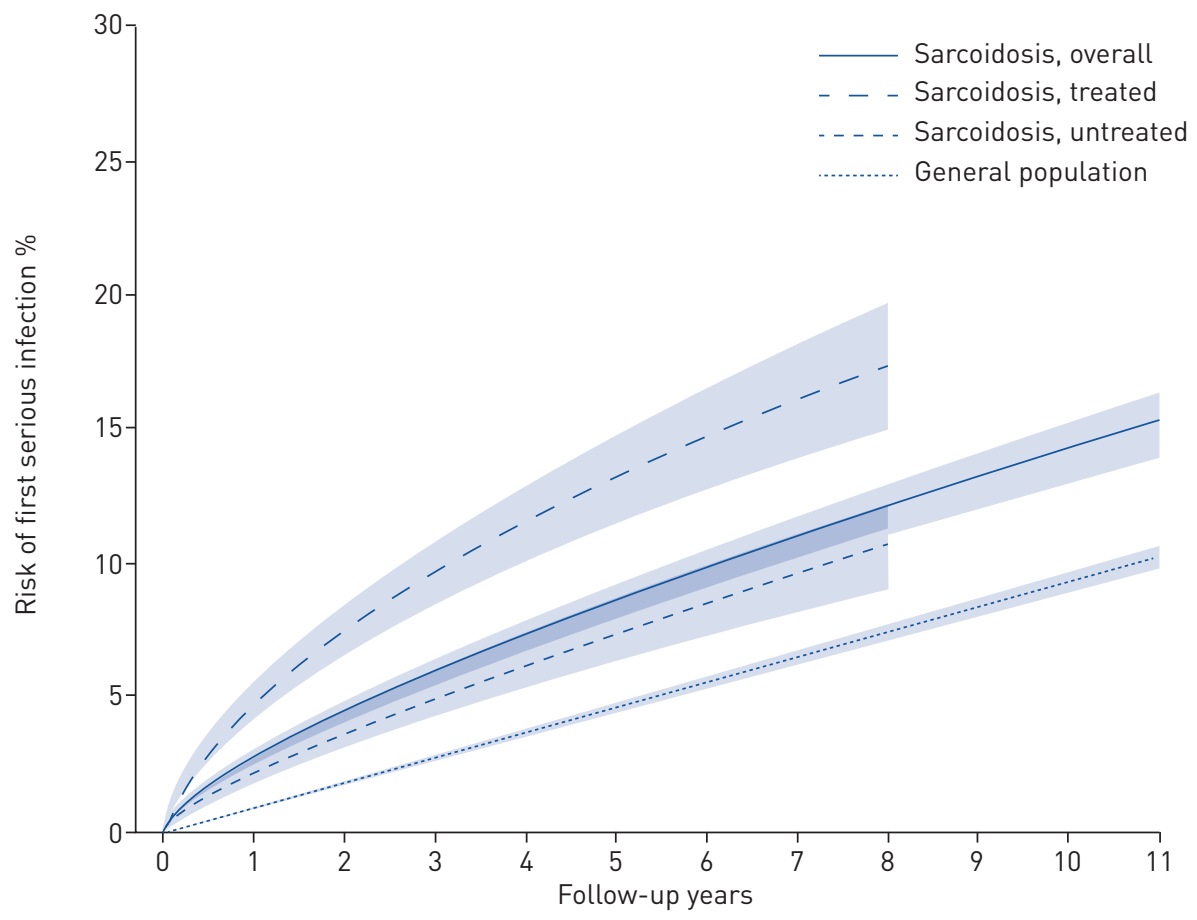

FIGURE 1 Risk of first serious infection in individuals with sarcoidosis loverall and stratified by treatment status around diagnosis) and their general population comparators. Treatment data were available for individuals with sarcoidosis entering the cohort starting 2006, hence follow-up for those was shorter than overall sarcoidosis and the general population comparators.

ours. We additionally found a slightly higher aHR for serious infection in females (2.0) than in males (1.6), but no differences by age at diagnosis.

Two key factors are likely to explain the higher risk for first and recurrent serious infection in sarcoidosis, especially the spike in serious infections during the first 2 years after sarcoidosis diagnosis. During this time, patients are under frequent follow-up and $\sim 40 \%$ receive immunosuppressants [10]. As indicated in sensitivity analyses and previous research [12], the peak in serious infection risk could be partly explained

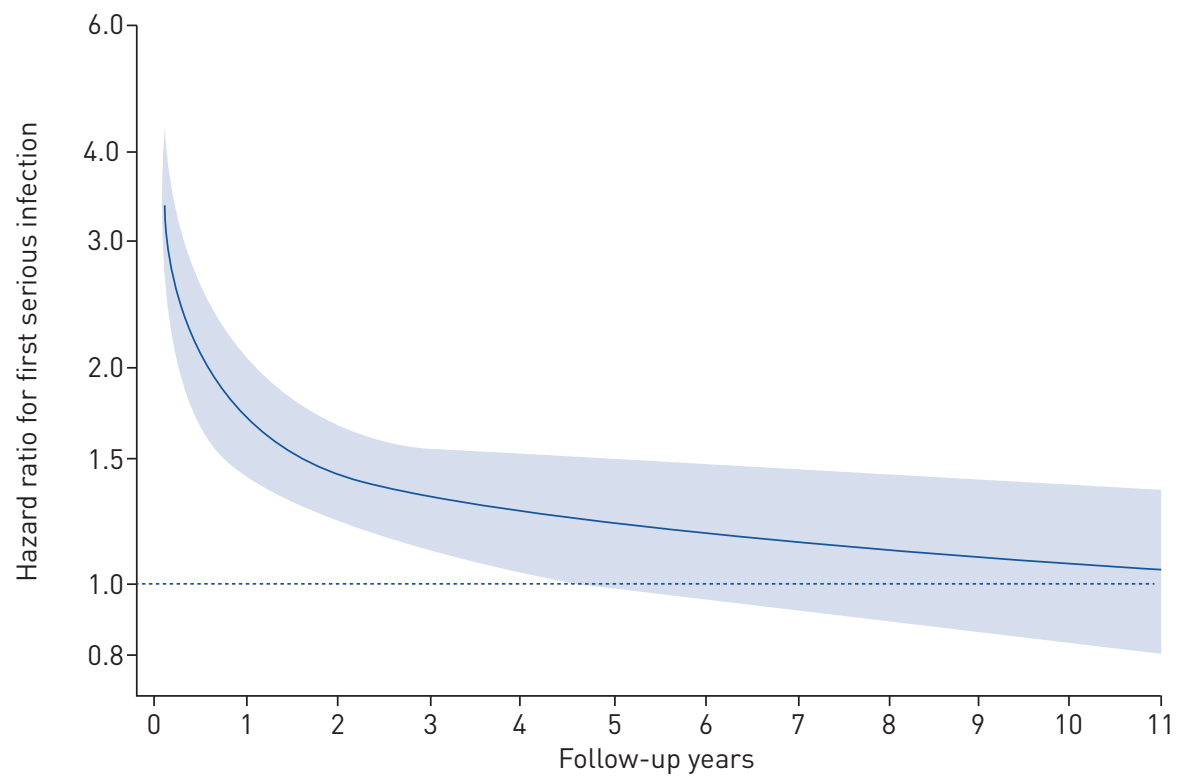

FIGURE 2 Adjusted hazard ratios for first serious infection by follow-up years comparing individuals with sarcoidosis to the general population. 
TABLE 3 Proportion of individuals and median time to recurrent serious infection and death, and hazard ratio for recurrent serious infection comparing individuals with sarcoidosis to the general population

\begin{tabular}{|c|c|c|c|c|}
\hline & \multicolumn{2}{|c|}{ Sarcoidosis } & \multicolumn{2}{|c|}{ General population } \\
\hline & Subjects & $\begin{array}{c}\text { Years to } \\
\text { event }\end{array}$ & Subjects & $\begin{array}{l}\text { Years to } \\
\text { event }\end{array}$ \\
\hline Subjects & \multicolumn{2}{|c|}{8737} & \multicolumn{2}{|c|}{86376} \\
\hline \multicolumn{5}{|l|}{ Serious infection } \\
\hline 0 & $7841(89.7)$ & & 82493 (95.5) & \\
\hline 1 & $1224(14.0)$ & $1.9(0.6-4.1)$ & $6158(7.1)$ & $2.9(1.3-5.2)$ \\
\hline 2 & $489(5.6)$ & $2.9(1.3-5.0)$ & $1536(1.8)$ & $4.2(2.3-6.2)$ \\
\hline 3 & $248(2.8)$ & $3.8(1.6-5.5)$ & $696(0.8)$ & $4.9(2.7-6.4)$ \\
\hline 4 & 150 (1.7) & $4.4(2.6-5.7)$ & $305(0.4)$ & $5.4(3.2-6.9)$ \\
\hline 5 & $84(1.0)$ & $5.4(3.3-6.8)$ & $150(0.2)$ & $5.6(2.9-7.5)$ \\
\hline 6 & $105(1.2)$ & $6.2(4.2-7.4)$ & $224(0.3)$ & $5.2(3.6-7.5)$ \\
\hline \multicolumn{5}{|l|}{ Death } \\
\hline Overall & 539 (5.3) & $2.7(1.1-5.4)$ & 3010 (3.3) & $3.6(1.8-5.8)$ \\
\hline Due to serious infection ${ }^{\#}$ & $109(1.2)$ & & $499(0.6)$ & \\
\hline \multicolumn{5}{|c|}{$\begin{array}{l}\text { Hazard ratio for recurrent serious infection } \\
(95 \% \mathrm{Cl})\end{array}$} \\
\hline Crude & \multicolumn{2}{|c|}{$3.30(2.99-3.64)$} & \multicolumn{2}{|c|}{1.00 (referent) } \\
\hline Adjusted & \multicolumn{2}{|c|}{$2.79(2.51-3.10)$} & \multicolumn{2}{|c|}{1.00 (referent) } \\
\hline \multicolumn{5}{|c|}{$\begin{array}{l}\text { Data are presented as } \mathrm{n}, \mathrm{n}(\%) \text { or median (interquartile range), unless otherwise stated. }{ }^{\text {\# }} \text { : serious infection } \\
\text { coded as underlying or contributing cause of death on the death certificate (data from the Cause of Death } \\
\text { Register); }{ }^{\text {I: }} \text { within-individual hazard ratio estimated from a Cox proportional hazards model with a } \gamma \text {-frailty } \\
\text { term (per individual; a shared frailty model). Adjusted hazard ratio estimated using inverse probability of } \\
\text { sarcoidosis weights. }\end{array}$} \\
\hline
\end{tabular}

by the increased healthcare interaction, or possibly a lower threshold of hospitalisation for infection. However, our analyses suggested that initiation of immunosuppressants, particularly that of systemic corticosteroids, remains a major risk factor for serious infection in sarcoidosis.

In our study, individuals with sarcoidosis who were treated around diagnosis (almost all with corticosteroids) had a three-fold increased rate of serious infection, whereas those who were not had a $50 \%$ increased rate. Corticosteroids indisputably play some part in serious infection occurrence $[8,13,14]$. At the same time, we cannot rule out that sarcoid inflammation itself is responsible for some of the risk increase. Indeed, patients with sarcoidosis who do not require treatment around diagnosis are at a notably higher risk for serious infection than general population comparators. In addition, the decline in the rate of serious infection in sarcoidosis coincides with disease remission, which in $\sim 50 \%$ of patients is independent of treatment [7]. The pathophysiological mechanisms that could elucidate these complex phenomena have yet to be adequately understood. Possibly, sarcoid inflammation triggers a state of anergy towards pathogens [15] that is presumably amplified by immunosuppressant agents.

The rate of serious infection is lower in sarcoidosis than in some autoimmune diseases that have been investigated using similar data. Rates ranging from 12 to 39 per 1000 person-years were reported in rheumatoid arthritis [16-18] and up to 100 per 1000 person-years in lupus [5, 20]. Relative to the general population, these rates vary from an increase of approximately two-fold in rheumatoid arthritis [16-19] to up to 10-fold higher in lupus [20]. In other respiratory diseases, the aHR for serious infection was lower in asthma than in sarcoidosis (aHR 1.5) [21], whereas in COPD a much higher aHR of 5.0 was reported [22]. Because differences partly depend on distribution of factors that vary amongst diseases (e.g. age, sex, treatment and study period), they should be interpreted with caution.

Individuals with sarcoidosis are more likely to be hospitalised for multiple serious infections. True risks of recurrence of serious infection are possibly higher than reported considering that premature death, which is more likely in sarcoidosis, prevents serious infections from recurring. Because recurrence has a negative impact on quality of life and is an indicator of high mortality $[1,2,4]$, future investigations are warranted to identify patients at risk for recurrence that could benefit from preventive interventions.

There are several limitations to our study, of which misclassification of serious infection is the most prominent, because serious infections could not be validated using microbiological examination results. Serious infection misclassification was more probable in sarcoidosis since one in four serious infections 
TABLE 4 Rates and hazard ratios for first serious infection comparing sarcoidosis to the general population in sensitivity analyses

\begin{tabular}{|c|c|c|c|c|c|}
\hline & $\begin{array}{l}\text { Individuals } \\
\text { at risk }\end{array}$ & Events & $\begin{array}{c}\text { Adjusted rate per } \\
1000 \text { person-years } \\
\text { (95\% Cl) }\end{array}$ & $\begin{array}{l}\text { Hazard ratio }{ }^{\#} \\
\text { (95\% CI) }\end{array}$ & $\begin{array}{l}\text { Adjusted hazard } \\
\text { ratio" }(95 \% \mathrm{Cl})\end{array}$ \\
\hline \multicolumn{6}{|c|}{ Hospitalisation and $\geqslant 1$ dispensation of an antimicrobial \pm 15 days from hospital admission $\pi$} \\
\hline Sarcoidosis & 6723 & 427 & $14.7(13.0-16.6)$ & $2.82(2.53-3.14)$ & $2.23(1.96-2.54)$ \\
\hline General population & 66441 & 1562 & $6.6(6.3-6.9)$ & 1.00 (Referent) & 1.00 (Referent) \\
\hline \multicolumn{6}{|c|}{ Excluding pneumonias from serious infection definition } \\
\hline Sarcoidosis & 8737 & 694 & $13.1(11.9-14.4)$ & $2.32(2.14-2.52)$ & $1.72(1.56-1.90)$ \\
\hline General population & 86376 & 3088 & $7.6(7.3-7.9)$ & 1.00 (Referent) & 1.00 (Referent) \\
\hline \multicolumn{6}{|c|}{ Excluding urinary tract infections from serious infection definition } \\
\hline Sarcoidosis & 8737 & 866 & $16.8(15.4-18.3)$ & $2.51(2.33-2.70)$ & $1.89(1.72-2.07)$ \\
\hline General population & 86376 & 3600 & $8.9(8.6-9.2)$ & 1.00 (Referent) & 1.00 (Referent) \\
\hline \multicolumn{6}{|c|}{$\begin{array}{l}\text { Requiring comparators to have } \geqslant 1 \text { healthcare visit in the NPR within } 2 \text { years before first visit for } \\
\text { sarcoidosis or corresponding date in comparators }\end{array}$} \\
\hline Sarcoidosis, overall & 8737 & 895 & $17.4(16.0-18.9)$ & $1.59(1.48-1.72)$ & $1.23(1.12-1.35)$ \\
\hline General population & 42406 & 2704 & $14.2(13.6-14.7)$ & 1.00 (Referent) & 1.00 (Referent) \\
\hline Sarcoidosis, treated" & 2762 & 326 & $29.8(25.9-34.3)$ & $2.45(2.15-2.80)$ & $2.08(1.77-2.43)$ \\
\hline General population & 13852 & 696 & $14.4(13.3-15.5)$ & 1.00 (Referent) & 1.00 (Referent) \\
\hline Sarcoidosis, not treated & 3961 & 275 & $15.7(13.5-18.3)$ & $1.28(1.12-1.46)$ & $1.03(0.88-1.22)$ \\
\hline General population" & 19802 & 1054 & $15.2(14.3-16.2)$ & 1.00 (Referent) & 1.00 (Referent) \\
\hline \multicolumn{6}{|c|}{$\begin{array}{l}\text { Excluding individuals with a serious infection within a year before first visit for sarcoidosis or } \\
\text { corresponding date in comparators }\end{array}$} \\
\hline Sarcoidosis & 8396 & 802 & $16.3(15.0-17.9)$ & $2.30(2.13-2.49)$ & $1.77(1.61-1.95)$ \\
\hline General population & 85678 & 3716 & $9.2(8.9-9.5)$ & 1.00 (Referent) & 1.00 (Referent) \\
\hline \multicolumn{6}{|l|}{ In Karolinska clinical cohort } \\
\hline Sarcoidosis & 693 & 47 & $11.1(7.8-15.9)$ & $1.80(1.32-2.46)$ & $1.49(1.02-2.18)$ \\
\hline General population & 6867 & 261 & $7.4(6.6-8.4)$ & 1.00 (Referent) & 1.00 (Referent) \\
\hline
\end{tabular}

Data are presented as $\mathrm{n}$, unless otherwise stated. NPR: National Patient Register. ${ }^{\#}$ : adjusted rates and adjusted hazard ratios were estimated using Poisson or Cox regression models weighted for inverse probability of sarcoidosis weights and years of follow-up as the time scale. Hazard ratios obtained from a cohort matched on age, sex and residential location; ף: ascertained in individuals included starting January 1, 2006 for whom medication dispensations could be obtained from the Prescribed Drug Register. 
was a pneumonia, which may share a similar clinical picture with the disease. To address this limitation, we used a strict definition requiring serious infection to be the primary discharge diagnosis, excluded all pneumonias or required a dispensation of an antimicrobial in addition to the hospital admission. These changes in serious infection definition had little effect on our results.

Furthermore, we did not have information on lifestyle factors (e.g. smoking) or vaccination rates among sarcoidosis cases and comparators. Based on previously published bias simulations for smoking [4], we expect that the true association between sarcoidosis and serious infection is somewhat stronger than the one reported here. Similarly, if individuals with sarcoidosis were more likely to be vaccinated, the observed findings may reflect an underestimation of the truth.

Due to the lack of detailed clinical information in the NPR, it is likely that there was some misclassification of sarcoidosis. However, preliminary validation data suggest that the accuracy of our definition is high ( positive predictive value $>90 \%$; unpublished data), and as we have shown previously $[4,23]$, our results are robust to nondifferential misclassification of sarcoidosis. In addition, we observed an association in the Karolinska sarcoidosis cohort despite small numbers and differences in serious infection predictors at baseline (e.g. age and treatment status; data not shown). We stratified our analyses by treatment status around diagnosis, but there were other comorbidities such as autoimmune diseases that were more prevalent in sarcoidosis than in the general population, which may also modify the risk. In a post hoc analysis, we found similar results stratifying by history of autoimmune disease (aHR for serious infection, 1.7 for history of autoimmune disease versus 1.8 for no history). Lastly, in the absence of a severity index for sarcoidosis that is independent of treatment, we could not distinguish between serious infections caused by treatment or sarcoid inflammation.

Nonetheless, by using longitudinal register data we were able to obtain a large and unselected sarcoidosis population, study recurrent serious infections and eliminate losses to follow-up. We expect our findings to be generalisable to populations in which modifiers of serious infection risk, such as healthcare standards, thresholds for hospitalisation, vaccination and treatment patterns are similar to those in Sweden.

In summary, sarcoidosis is associated with a higher risk of serious infection compared to the general population, especially during the first 2 years after diagnosis. Individuals treated around the time of sarcoidosis diagnosis, probably due to severe symptoms or impaired organ function, are at noticeably higher risk of serious infection. Although we cannot withhold pharmacological treatment in patients who need it, we should further examine which measures such as vaccinations, closer follow-up or prescription of steroid-sparing medications could alleviate the excess risk of serious infection in these patients.

Author contributions: M. Rossides: conceptualisation, data curation, formal analysis, methodology, software, visualisation, writing (original draft), writing (review and editing); S. Kullberg: writing (review and editing); A. Eklund: writing (review and editing); D. Di Giuseppe: writing (review and editing); J. Grunewald: writing (review and editing); J. Askling: conceptualisation, writing (review and editing); E.V. Arkema: conceptualisation, funding acquisition, resources, supervision, writing (review and editing).

Conflict of interest: M. Rossides has nothing to disclose. S. Kullberg has nothing to disclose. A. Eklund has nothing to disclose. D. Di Giuseppe has nothing to disclose. J. Grunewald reports grants from Swedish Heart-Lung Foundation (Hjärt-Lungfonden; grant number 20190478), during the conduct of the study. J. Askling has nothing to disclose. E.V. Arkema reports grants from Swedish Heart-Lung Foundation (Hjärt-Lungfonden; grant number 20170412), during the conduct of the study. M. Rossides has nothing to disclose.

Support statement: The study was supported by a grant from the Swedish Heart-Lung Foundation (Hjärt-Lungfonden; project number 20170412 and 20190478). The data linkage used in this study was funded by a grant from the Swedish Society of Medicine (Svenska Läkaresällskapet). Sarcoidosis research at Karolinska Institutet is also supported by the Swedish Research Council (Vetenskapsrådet), the Strategic Research Area in Epidemiology at Karolinska Institutet (SfoEpi), and by a regional agreement on medical training and clinical research (ALF) between Region Stockholm and Karolinska Institutet. Funding information for this article has been deposited with the Crossref Funder Registry.

\section{References}

1 Pick HJ, Bolton CE, Lim WS, et al. Patient-reported outcome measures in the recovery of adults hospitalised with community-acquired pneumonia: a systematic review. Eur Respir J 2019; 53: 1802165.

2 Tong S, Amand C, Kieffer A, et al. Trends in healthcare utilization and costs associated with pneumonia in the United States during 2008-2014. BMC Health Serv Res 2018; $18: 715$.

3 Falasinnu T, Rossides M, Chaichian Y, et al. Do death certificates underestimate the burden of rare diseases? The example of systemic lupus erythematosus mortality, Sweden, 2001-2013. Public Health Rep 2018; 133: 481-488.

4 Rossides M, Kullberg S, Askling J, et al. Sarcoidosis mortality in Sweden: a population-based cohort study. Eur Respir J 2018; 51: 1701815.

5 Feldman CH, Hiraki LT, Winkelmayer WC, et al. Serious infections among adult Medicaid beneficiaries with systemic lupus erythematosus and lupus nephritis. Arthritis Rheumatol 2015; 67: 1577-1585.

6 Singh JA, Cameron C, Noorbaloochi S, et al. Risk of serious infection in biological treatment of patients with rheumatoid arthritis: a systematic review and meta-analysis. Lancet 2015; 386: 258-265. 
Grunewald J, Grutters JC, Arkema E V, et al. Sarcoidosis. Nat Rev Dis Primers 2019; 5: 45.

8 Ungprasert P, Crowson CS, Matteson EL. Sarcoidosis increases risk of hospitalized infection. A population-based study, 1976-2013. Ann Am Thorac Soc 2017; 14: 676-681.

9 Socialstyrelsen. Kvalitet och innehåll i patientregistret. Utskrivningar från slutenvården 1964-2007 och besök i specialiserad öppenvård (exklusive primärvårdsbesök) 1997-2007 [Quality and contents of the National Patient Register]. Stockholm, Socialstyrelsen, 2009.

10 Rossides M, Kullberg S, Eklund A, et al. Sarcoidosis diagnosis and treatment in Sweden: a register-based assessment of variations by region and calendar period. Respir Med 2020; 161: 105846.

11 Royston P, Lambert PC. Flexible Parametric Survival Analysis Using Stata: Beyond the Cox Model. College Station, TX, Stata Press, 2011.

12 Rossides M, Kullberg S, Askling J, et al. Are infectious diseases risk factors for sarcoidosis or a result of reverse causation? Findings from a population-based nested case-control study. Eur J Epidemiol 2020; in press [https://10. 1007/s10654-020-00611-w].

13 Dixon WG, Abrahamowicz M, Beauchamp M-E, et al. Immediate and delayed impact of oral glucocorticoid therapy on risk of serious infection in older patients with rheumatoid arthritis: a nested case-control analysis. Ann Rheum Dis 2012; 71: 1128-1133.

14 Waljee AK, Rogers MAM, Lin P, et al. Short term use of oral corticosteroids and related harms among adults in the United States: population based cohort study. BMJ 2017; 357: j1415.

15 Miyara M, Amoura Z, Parizot C, et al. The immune paradox of sarcoidosis and regulatory T cells. J Exp Med 2006; 203: 359-370.

16 Franklin J, Lunt M, Bunn D, et al. Risk and predictors of infection leading to hospitalisation in a large primary-care-derived cohort of patients with inflammatory polyarthritis. Ann Rheum Dis 2007; 66: 308-312.

17 Smitten AL, Choi HK, Hochberg MC, et al. The risk of hospitalized infection in patients with rheumatoid arthritis. J Rheumatol 2008; 35: 387-393.

18 Widdifield J, Bernatsky S, Paterson JM, et al. Serious infections in a population-based cohort of 86,039 seniors with rheumatoid arthritis. Arthritis Care Res 2013; 65: 353-361.

19 Listing J, Gerhold K, Zink A. The risk of infections associated with rheumatoid arthritis, with its comorbidity and treatment. Rheumatology 2013; 52: 53-61.

20 Tektonidou MG, Wang Z, Dasgupta A, et al. Burden of serious infections in adults with systemic lupus erythematosus: a national population-based study, 1996-2011. Arthritis Care Res 2015; 67: 1078-1085.

21 Helby J, Nordestgaard BG, Benfield T, et al. Asthma, other atopic conditions and risk of infections in 105519 general population never and ever smokers. J Intern Med 2017; 282: 254-267.

22 Inghammar M, Engström G, Ljungberg B, et al. Increased incidence of invasive bacterial disease in chronic obstructive pulmonary disease compared to the general population - a population based cohort study. BMC Infect Dis 2014; 14: 163.

23 Rossides M, Grunewald J, Eklund A, et al. Familial aggregation and heritability of sarcoidosis: a Swedish nested case-control study. Eur Respir J 2018; 52: 1800385 